\title{
Cytokeratin 7 and 20 Expression in Choroid Plexus Tumors: Utility in Differentiating These Neoplasms from Metastatic Carcinomas
}

\author{
Kymberly A. Gyure, M.D., Alan L. Morrison, M.D. \\ University of Maryland Medical System (KAG), Baltimore, Maryland, and Armed Forces Institute of \\ Pathology (KAG, ALM), Washington, D.C.
}

Tumors derived from choroid plexus epithelium are uncommon and may exhibit a wide variety of histologic patterns. They often are difficult to distinguish from metastatic carcinomas. Previous studies that addressed this issue yielded conflicting results. Recent reports have demonstrated that evaluation of coordinate expression of cytokeratin (CK) 7 and CK20 aids in distinguishing primary from metastatic lesions in a number of anatomic sites and that tumors that commonly are metastatic to the brain retain their CK7/CK20 immunophenotype in this location. We examined 35 choroid plexus tumors with a panel of antibodies to determine their CK7/ CK20 immunophenotype. Tumors from 35 patients (7 male, 28 female; mean age, 25 years), including 31 choroid plexus papillomas and 4 atypical papillomas, were evaluated. All tumors were intraventricular or within the cerebellopontine angle and composed predominantly of orderly columnar epithelial cells resting on distinct fibrovascular cores. Atypical papillomas contained combinations of focal loss of architectural pattern, increased mitotic activity, necrosis, and brain parenchymal invasion. No lesion was unequivocally malignant. Twenty-six tumors (74\%), including all atypical papillomas, were CK7 positive and CK20 negative. Two tumors stained with both markers, one stained with CK20 only, and six stained with neither marker. Other findings included expression of glial fibrillary acidic protein in 24 tumors, S-100 protein in 19 tumors, transthyretin in 31 tumors, Ber EP4 in 1 tumor, CAM5.2 in 33 tumors, epithelial membrane antigen in 4 tumors, and pancytokeratin in 27 tumors. Our results indicate that the majority of choroid plexus

Copyright (C) 2000 by The United States and Canadian Academy of Pathology, Inc.

VOL. 13, NO. 6, P. 638, 2000 Printed in the U.S.A

Date of acceptance: December 8, 1999.

Presented in part at the 75th annual meeting of the American Association of Neuropathologists, Portland, Oregon, June 18, 1999.

Address reprint requests to: Kymberly A. Gyure, M.D., Department of Pathology, Room NBW43, University of Maryland Medical System, 22 South Greene Street, Baltimore, MD 21201. tumors have a CK7-positive/CK20-negative immunophenotype. This finding may be useful in differentiating these lesions from metastatic carcinomas that have differing CK7/CK20 profiles.

KEY WORDS: Choroid plexus tumors, Cytokeratin 7, Cytokeratin 20, Immunohistochemistry.

Mod Pathol 2000;13(6):638-643

Choroid plexus tumors are relatively rare lesions, accounting for fewer than $1 \%$ of intracranial neoplasms $(1,2)$. They may exhibit a wide spectrum of histologic patterns, including acinar $(3,4)$, mucussecreting $(5,6)$, oncocytic $(7,8)$, pigmented $(9,10)$, tubular (11), and metaplastic $(5,12,13)$ variants. Distinguishing these tumors from metastatic carcinomas, a matter of considerable therapeutic and prognostic significance, therefore can be problematic. A number of potential immunohistochemical markers for choroid plexus tumors, including glial fibrillary acidic protein (14-25), S-100 protein (14$17,21,23)$, vimentin (14-17), cytokeratin (CK) (14$20,22)$, non-CK epithelial markers $(16,17,21,26)$, carcinoembryonic antigen $(14,15,18,21)$, neuronal markers $(15,21,27)$, and transthyretin $(14,21,23$, $28,29)$, have been evaluated; however, their utility has been limited by a lack of specificity and by the conflicting results yielded by these studies.

Recently, the evaluation of the coordinate expression of CK7 and CK20 has been shown to be of use in distinguishing primary from metastatic lesions in a number of anatomic sites (30-32). The CK7/CK20 immunophenotypes of a large number of neoplasms have been characterized (Table 1). Moreover, it has been shown that these lesions retain their CK7/CK20 immunophenotype in metastatic locations, including the brain (33).

We examined 35 choroid plexus tumors with a panel of antibodies, including CK7 and CK20, to determine their immunophenotype. The potential diagnostic implications of these findings are discussed. 
TABLE 1. Summary of Predominant CK7/CK20 Immunophenotypes of Various Tumors (32, 36-39)

\begin{tabular}{cl}
\hline Immunophenotype & \multicolumn{1}{c}{ Tumor } \\
\hline $\mathrm{CK}^{+} / \mathrm{CK} 20^{+}$ & Cholangiocarcinoma/biliary duct carcinoma $^{a}$ \\
& Gastric adenocarcinoma ${ }^{a}$ \\
& Ovarian mucinous carcinoma \\
& Pancreatic adenocarcinoma \\
& Transitional cell carcinoma \\
& Ductal and lobular breast carcinoma \\
& Malignant mesothelioma \\
& Endometrial adenocarcinoma \\
& Ovarian serous and endometrioid carcinoma \\
& Pulmonary adenocarcinoma/bronchioalveolar \\
& carcinoma \\
& Colorectal adenocarcinoma \\
& Gastric adenocarcinoma ${ }^{-}$ \\
& Merkel cell carcinoma \\
& Hepatocellular carcinoma \\
& Prostatic adenocarcinoma \\
& Renal cell carcinoma \\
& Small cell carcinoma of lung \\
& Squamous cell carcinoma of esophagus, lung, \\
& skin \\
& Thyroid carcinoma (follicular and papillary) \\
& \\
&
\end{tabular}

${ }^{a}$ Variable.

\section{MATERIALS AND METHODS}

All cases diagnosed as choroid plexus papilloma or carcinoma at the Armed Forces Institute of Pathology between 1955 and 1998 in which sufficient paraffin-embedded material was available were reviewed. Hematoxylin and eosin-stained sections, as well as histochemically and immunohistochemically stained sections when performed, were reviewed in all cases. Lesions were classified as choroid plexus papilloma, atypical choroid plexus papilloma, or choroid plexus carcinoma using recent diagnostic criteria (34). Clinical information, including the age and sex of the patient, the location of the lesion, and a history of previous malignancy, was reviewed and tabulated when present as part of the information submitted with the case. Patients in whom a metastatic lesion could not be excluded based on the clinical data and pathologic findings were excluded from the study, leaving a total of 35 cases.

Sections were prepared from a single representative paraffin block in each case and were stained with antibodies to Ber EP4 (Dako, Carpinteria, CA; dilution 1:50), CAM 5.2 (Becton Dickinson Inc., San Jose, CA; dilution 1:50), CK7 (OV-TL 12/30; Dako; dilution 1:400), CK20 (Ks 20.8, Dako; dilution 1:100), epithelial membrane antigen (EMA; E29; Dako; dilution 1:100), glial fibrillary acidic protein (GFAP; Dako; dilution 1:2000), a pancytokeratin cocktail (AE1/3/LP34; Boehringer Mannheim, Indianapolis, IN; Dako; dilution 1:200/1:40), S-100 protein (Dako; dilution 1:400), and transthyretin (prealbumin; Dako; dilution 1:640) using a standard avidinbiotinylated peroxidase method (35). For the monoclonal antibodies (Ber EP4, CAM 5.2, CK7,
CK20, EMA, and the pancytokeratin cocktail) and for GFAP, antigen retrieval with protease was performed (35). Each antibody stain was interpreted as positive or negative, and, when positive, a semiquantitative estimate of the number of positive cells was determined as follows: 1 to $25 \%$ positive cells, $1+; 26$ to $50 \%$ positive cells, $2+$; 51 to $75 \%$ positive cells, $3+; 76$ to $100 \%$ positive cells, $4+$; equivocal or weak staining, $+/-$. Only staining within epithelial cells was considered to be positive.

\section{RESULTS}

The clinical and immunohistochemical results are summarized in Table 2. Tumors in 7 males and 28 females ranging in age from 3 months to 65 years (mean age, 25 years) were evaluated. All lesions were either intraventricular or within the cerebellopontine angle (CPA): 8 lesions were lateral ventricular tumors, 2 were third ventricular tumors, 23 were fourth ventricular/posterior fossa tumors, and 2 were CPA tumors.

All choroid plexus tumors were composed predominantly of orderly columnar epithelial cells resting on distinct fibrovascular cores (Fig. 1). Occasional lesions exhibited foci of acinar (eight tumors [Fig. 2]) or oncocytic (one tumor) differentiation. In tumors classified as typical choroid plexus papillomas, mitotic activity generally was inconspicuous ( $\leq 6$ mitoses per 10 high-power fields in all cases) and necrosis (one tumor), microscopic foci of brain invasion (four tumors), and small areas with a loss of papillary architecture (four tumors) were rare findings. Four lesions contained sufficient mitotic activity (up to 12 mitoses per 10 high-power fields) and combinations of necrosis, brain invasion, and loss of architecture to warrant classification as atypical papillomas (Fig. 3). No lesion was unequivocally malignant.

Most tumors $(80 \%)$, including all four atypical choroid plexus papillomas, exhibited strong cytoplasmic staining in a variable number of cells when stained with anti-CK7 antibodies (Fig. 4). All but two of these lesions (74\%) were CK20 negative. One papilloma stained with CK20 only in fewer than $25 \%$ of the cells, and six tumors failed to stain with either marker. There was no difference in either the intensity of staining or the number of positive cells between classic choroid plexus papillomas and atypical papillomas.

Other antibodies staining the majority of choroid plexus tumors included those directed against CAM 5.2 (positive in 94\%), pancytokeratin (positive in $77 \%$ ), GFAP (positive in $69 \%$ ), S-100 protein (positive in 54\%), and transthyretin (positive in $89 \%$ ). There were no significant differences in staining between papillomas and atypical papillomas with these markers. 
TABLE 2. Summary of Clinical and Immunohistochemical Findings in 35 Choroid Plexus Tumors

\begin{tabular}{|c|c|c|c|c|c|c|c|c|c|c|c|}
\hline Age $(y) / \operatorname{sex}$ & Location & Diagnosis & Ber EP4 & CAM 5.2 & CK7 & CK20 & EMA & GFAP & Pancytokeratin & $\begin{array}{c}\text { S-100 } \\
\text { Protein }\end{array}$ & Transthyretin \\
\hline $3 \mathrm{mo} / \mathrm{male}$ & Left lateral ventricle & $\mathrm{CPP}$ & - & - & $1+$ & - & - & - & - & $+1-$ & $4+$ \\
\hline $1 /$ male & Lateral ventricle & $\mathrm{CPP}$ & - & $4+$ & - & - & - & $4+$ & $4+$ & - & $4+$ \\
\hline $1 /$ female & Left lateral ventricle & CPP & - & $3+$ & $2+$ & - & - & $2+$ & $2+$ & $1+$ & $1+$ \\
\hline $2 /$ female & 3rd ventricle & CPP & - & $2+$ & - & - & - & $3+$ & $4+$ & $2+$ & $1+$ \\
\hline $3 /$ female & Right lateral ventricle & $\mathrm{CPP}$ & - & - & - & - & - & - & $1+$ & $+1-$ & $4+$ \\
\hline 6/female & Left lateral ventricle & $\mathrm{CPP}$ & - & $1+$ & $1+$ & - & - & - & - & - & $2+$ \\
\hline 6/female & Posterior fossa & СРP & - & $1+$ & $1+$ & - & - & - & - & - & $4+$ \\
\hline 6/female & 4 th ventricle & $\mathrm{CPP}$ & - & $3+$ & $2+$ & - & - & $1+$ & $2+$ & $+1-$ & $+1-$ \\
\hline $8 /$ male & 4 th ventricle & CPP & $+1-$ & $2+$ & $2+$ & - & - & $1+$ & $1+$ & - & $3+$ \\
\hline 14/female & 4 th ventricle & $\mathrm{CPP}$ & - & $4+$ & - & - & - & $4+$ & $3+$ & $4+$ & $4+$ \\
\hline $17 /$ female & Posterior fossa & CPP & - & $4+$ & $1+$ & - & $+1-$ & $1+$ & $1+$ & $4+$ & $2+$ \\
\hline 20/female & Posterior fossa & CPP & $+1-$ & $4+$ & $2+$ & $3+$ & $2+$ & $4+$ & $4+$ & $4+$ & $2+$ \\
\hline $22 /$ female & 4th ventricle & CPP & - & $1+$ & $1+$ & - & - & $2+$ & $1+$ & - & $4+$ \\
\hline $23 /$ male & Posterior fossa & $\mathrm{CPP}$ & - & $2+$ & $1+$ & - & - & - & - & - & $2+$ \\
\hline 25/female & 4 th ventricle & $\mathrm{CPP}$ & - & $4+$ & $1+$ & - & - & $+1-$ & - & - & $3+$ \\
\hline $25 /$ female & 4 th ventricle & CPP & - & $4+$ & $3+$ & - & - & - & $4+$ & $4+$ & $4+$ \\
\hline 26/female & 4 th ventricle & CPP & - & $3+$ & $2+$ & - & - & $1+$ & $2+$ & $4+$ & $4+$ \\
\hline 26/female & Posterior fossa & CPP & - & $3+$ & $2+$ & - & - & $1+$ & $1+$ & $+1-$ & $2+$ \\
\hline $30 /$ female & Right CP angle & CPP & - & $1+$ & $1+$ & - & - & - & - & $4+$ & $4+$ \\
\hline $31 /$ female & 4 th ventricle & $\mathrm{CPP}$ & - & $4+$ & $3+$ & - & - & $4+$ & $1+$ & - & $4+$ \\
\hline $32 /$ female & 4 th ventricle & $\mathrm{CPP}$ & - & $4+$ & $2+$ & - & - & $+1-$ & $2+$ & $4+$ & $3+$ \\
\hline $34 /$ male & Left CP angle & $\mathrm{CPP}$ & - & $4+$ & - & $1+$ & $1+$ & $4+$ & $1+$ & $3+$ & $3+$ \\
\hline $37 /$ female & Right lateral ventricle & $\mathrm{CPP}$ & - & $4+$ & $2+$ & - & - & $+1-$ & $2+$ & $4+$ & - \\
\hline 39/female & 4 th ventricle & $\mathrm{CPP}$ & - & $4+$ & - & - & - & - & $2+$ & $4+$ & $+1-$ \\
\hline 39/female & Posterior fossa & CPP & - & $3+$ & $1+$ & - & - & $4+$ & $1+$ & $4+$ & $2+$ \\
\hline $40 /$ female & 4 th ventricle & $\mathrm{CPP}$ & - & $4+$ & $2+$ & - & - & $4+$ & $1+$ & $4+$ & $4+$ \\
\hline 45/female & 4 th ventricle & CPP & - & $4+$ & $1+$ & - & $1+$ & $4+$ & $3+$ & $4+$ & $4+$ \\
\hline $51 /$ female & 4 th ventricle & CPP & - & $2+$ & - & - & - & $1+$ & $3+$ & - & $4+$ \\
\hline $55 /$ female & Posterior fossa & CPP & - & $1+$ & $1+$ & - & - & $3+$ & $+1-$ & $+1-$ & $2+$ \\
\hline $61 /$ female & 4 th ventricle & CPP & $1+$ & $4+$ & $4+$ & $3+$ & - & $2+$ & $4+$ & $4+$ & $4+$ \\
\hline $65 /$ female & 4 th ventricle & $\mathrm{CPP}$ & - & $3+$ & $1+$ & - & $+1-$ & $3+$ & - & $3+$ & $3+$ \\
\hline $5 \mathrm{mo} / \mathrm{male}$ & 3rd ventricle & АCРP & - & $1+$ & $1+$ & - & $+1-$ & $1+$ & $1+$ & - & $2+$ \\
\hline 4/female & Right lateral ventricle & ACPP & - & $1+$ & $1+$ & - & $1+$ & $1+$ & $4+$ & - & $3+$ \\
\hline $32 /$ male & 4 th ventricle & ACPP & - & $4+$ & $2+$ & - & - & $4+$ & $3+$ & $4+$ & $2+$ \\
\hline 33/female & Left lateral ventricle & ACPP & $+1-$ & $4+$ & $4+$ & - & - & $1+$ & $4+$ & $2+$ & $+1-$ \\
\hline
\end{tabular}

EMA, epithelial membrane antigen; GFAP, glial fibrillary acidic protein; CP, cerebellopontine angle; CPP, choroid plexus papilloma; ACPP, atypical choroid plexus papilloma.

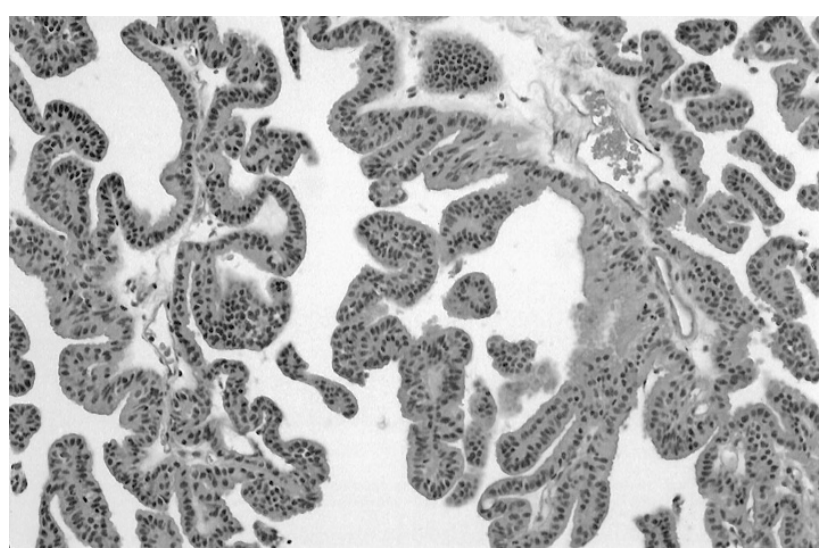

FIGURE 1. Classic choroid plexus papillomas were composed predominantly of a single layer of cuboidal to columnar epithelial cells resting on delicate fibrovascular cores (hematoxylin and eosin; original magnification, $100 \times)$.

Markers that were negative in the vast majority of choroid plexus tumors included EMA (positive in $11 \%$ ) and Ber EP4 (positive in 3\%). It is interesting that the single Ber EP4-positive lesion was also CK20 positive and exhibited foci of acinar differentiation histologically. Two of the EMA-positive neoplasms also were CK20 positive.

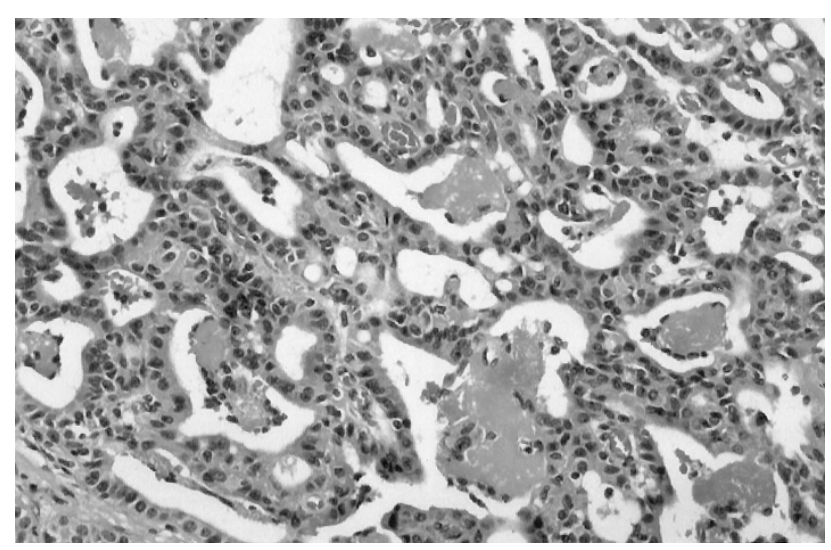

FIGURE 2. Several papillomas contained foci of glandular/acinar differentiation (hematoxylin and eosin; original magnification, 400×).

\section{DISCUSSION}

Distinguishing choroid plexus tumors from metastatic lesions, particularly in adult patients, may be extremely difficult. However, the prognostic and therapeutic implications of this distinction are significant. Therefore, a number of studies have examined a variety of markers to assist in this differential diagnosis; however, no single marker has proved to 


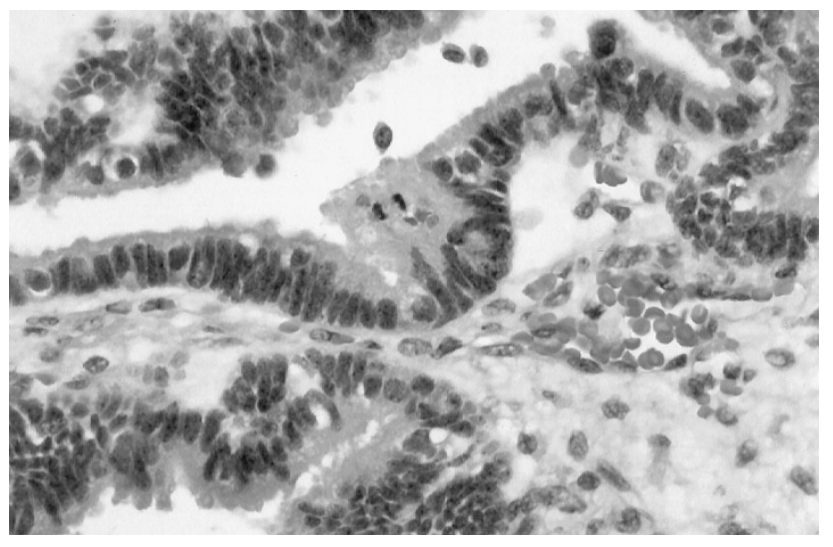

FIGURE 3. Atypical choroid plexus papillomas possessed areas of multilayered epithelium with moderate nuclear atypia and increased mitotic activity (hematoxylin and eosin; original magnification, 400×).

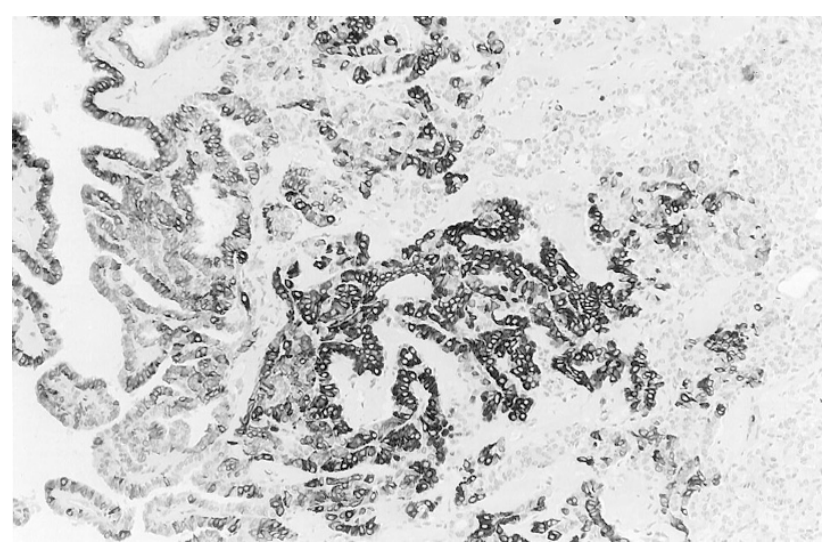

FIGURE 4. Most choroid plexus papillomas demonstrated strong expression of cytokeratin 7 in a variable number of epithelial cells (CK7 immunostain; original magnification, $200 \times$ ).

be sufficiently specific for routine clinical application (26).

Our findings confirm those of many others (14$25)$ that GFAP is expressed in a significant proportion of choroid plexus papillomas $(69 \%$ in our study). This has been interpreted as evidence of glial or ependymal differentiation in these lesions $(24,25)$ and is consistent with the origin of choroid plexus epithelium from primitive neuroepithelial cells. Because most metastatic carcinomas to the brain are GFAP negative (33), a positive finding is of some utility in this differential diagnosis.

S-100 protein is of limited differential diagnostic utility because it may be positive in metastatic lesions (26) and is negative in a significant proportion of choroid plexus tumors (nearly $50 \%$ in our series). It has been suggested that S-100 protein positivity predominates in well-differentiated neoplasms and therefore its absence may be a marker of malignancy in these lesions $(15,21,23)$. We were unable to confirm this observation; however, only a small number of atypical papillomas and no carcinomas were included in our study.
Transthyretin, a $55 \mathrm{kDa}$ protein that is involved in the transport of thyroxine and retinol and that is expressed in both normal and neoplastic choroid plexus epithelium, is considered to be the most useful marker to distinguish choroid plexus tumors from other papillary neoplasms (1). Our study confirms the high sensitivity of this marker for choroid plexus tumors (29). However, in some studies, a significant proportion of tumors failed to stain with antibodies to transthyretin $(21,28)$, and, more important, metastatic lesions may be positive when stained with this marker $(14,28)$. These findings limit the utility of transthyretin as a single marker in the differential diagnosis of choroid plexus neoplasms.

Non-CK epithelial markers also have been evaluated in choroid plexus tumors; of these, EMA has been the most widely studied $(16,17,21)$. Positivity with this marker has ranged from $11 \%$ of choroid plexus tumors (our series) to $71 \%$ (21). Reasons for this discrepancy are not clear; however, the wide range of positivity using this antibody among published studies limits its diagnostic usefulness. Among the other non-CK epithelial markers, HEA 125 and Ber EP4 show the most promise in differentiating choroid plexus tumors from metastatic lesions. Gottschalk and colleagues (26) demonstrated positive staining with these markers in 3 of 28 patients with choroid plexus tumors; in contrast, 44 of 45 metastatic lesions were positive. Our findings confirm the lack of Ber EP4 staining in choroid plexus tumors, and we concur with these authors' impression that Ber EP4 is a reliable tool in this differential diagnosis.

Finally, because choroid plexus tumors are composed predominantly of epithelial cells, the vast majority of these lesions express a variety of CK antigens $(14-20,22)$. Although this finding is of some help in distinguishing choroid plexus tumors from other primary central nervous system neoplasms $(14,20,22)$, it is of little use in differentiating choroid plexus neoplasms from metastatic carcinomas, the majority of which also express these antigens (33).

Recently, different subsets of CKs have been defined and found to be distributed in a tissuespecific manner (36). More important, a number of studies have shown that many carcinomas tend to recapitulate the CK subset profiles of the epithelium from which they arise and that the coordinate expression of CK subsets, in particular CK7 and CK20, may be useful in distinguishing primary from metastatic lesions in a variety of anatomic sites (30-32). CK7, a basic (type II) keratin, is found in the glandular epithelium of many organs, including lung, ovary, endometrium, and breast, and in adenocarcinomas that arise from these tissues (32, 36, 37). CK20, an acidic (type I) keratin, is expressed 
principally in epithelia and tumors derived from the gastrointestinal tract, urothelium, and Merkel cells $(32,36,38,39)$. To our knowledge, ours is the first study to evaluate CK7 and CK20 expression in choroid plexus tumors.

The majority of choroid plexus tumors in this study $(74 \%)$, including all atypical papillomas, possessed a CK7-positive/CK20-negative immunophenotype. Although this profile overlaps with those of several tumors that commonly are metastatic to the brain, including lung and breast carcinoma, several tumors that may have a predominantly papillary architecture and therefore be confused with choroid plexus tumors, including transitional cell carcinoma, colorectal adenocarcinoma, renal cell carcinoma, and papillary thyroid carcinoma, have differing CK7/CK20 immunophenotypes (Table 1). These findings suggest that CK7/CK20 immunophenotyping may have some utility in distinguishing choroid plexus tumors from metastatic lesions, particularly in patients who have known primary tumors with characteristic CK7/CK20 profiles. In cases in which the distinction between choroid plexus papilloma/carcinoma and a metastatic lesion cannot be definitively made on the basis of morphologic and immunohistochemical features, the CK7/CK20 immunophenotype may be helpful in directing the clinical workup for possible primary lesions.

In summary, our study demonstrates that the majority of choroid plexus tumors have a characteristic CK7-positive/CK20-negative immunophenotype. This finding may be useful in distinguishing these neoplasms from metastatic carcinomas that have differing CK7/CK20 profiles. Our findings also confirm the utility of GFAP, transthyretin, and Ber EP4 in this differential diagnosis. Used in combination, these markers should differentiate choroid plexus tumors from metastatic lesions in most cases.

Acknowledgments: The authors express their thanks to Robin-Anne V. Ferris, MFS, for expert photographic assistance, and to Denise Y. Young for her immunohistochemical expertise.

\section{REFERENCES}

1. Lantos PL, Vandenberg SR, Kleihues P. Tumors of the nervous system. In: Graham DI, Lantos PL, editors. Greenfield's neuropathology. 6th ed. London: Arnold; 1997. p. 647-53.

2. Surawicz TS, McCarthy BJ, Kupelian V, Jukich PJ, Bruner JM, Davis FG. Descriptive epidemiology of primary brain and CNS tumors: results from the Central Brain Tumor Registry of the United States, 1990-1994. Neuro-Oncology 1999;1:1425.

3. Ajir F, Chanbusarakum K, Bolles JC. Acinar choroid plexus adenoma of the fourth ventricle. Surg Neurol 1982;17:290-2.

4. Davis RL, Fox GE. Acinar choroid plexus adenoma: case report. J Neurosurg 1970;33:587-90.

5. Duckett S, Osterholm J, Schaefer D, Gonzales C, Schwartzman RJ. Ossified mucin-secreting choroid plexus adenoma: case report. Neurosurgery 1991;29:130-2.

6. Eljamel MSM, Jeffreys RV. Mucous-secreting choroid plexus adenoma-case report and review of the literature. Neuropediatrics 1990;21:55-6.

7. Diengdoh JV, Shaw MDD. Oncocytic variant of choroid plexus papilloma: evolution from benign to malignant "oncocytoma." Cancer 1993;71:855-8.

8. Stefanko SZ, Vuzevski VD. Oncocytic variant of choroid plexus papilloma. Acta Neuropathol 1985;66:160-2.

9. Reimund EL, Sitton JE, Harkin JC. Pigmented choroid plexus papilloma. Arch Pathol Lab Med 1990;114:902-5.

10. Boesel CP, Suhan JP. A pigmented choroid plexus carcinoma: histochemical and ultrastructural studies. J Neuropathol Exp Neurol 1979;38:177-86.

11. Andreini L, Doglioni C, Giangaspero F. Tubular adenoma of choroid plexus: a case report. Clin Neuropathol 1991;10:13740.

12. Cardozo J, Cepeda F, Quintero M, Mora E. Choroid plexus papilloma containing bone. Acta Neuropathol 1985;68:83-5.

13. Salazar J, Vaquero J, Aranda IF, Menèndez J, Jimenez MD, Bravo G. Choroid plexus papilloma with chondroma: case report. Neurosurgery 1986;18:781-3.

14. Ang LC, Taylor AR, Bergin D, Kaufmann JCE. An immunohistochemical study of papillary tumors in the central nervous system. Cancer 1990;65:2712-9.

15. Coffin CM, Wick MR, Braun JT, Dehner LP. Choroid plexus neoplasms: clinicopathologic and immunohistochemical studies. Am J Surg Pathol 1986;10:394-404.

16. Cruz-Sanchez FF, Rossi ML, Hughes JT, Coakham HB, Figols J, Eynaud PM. Choroid plexus papillomas: an immunohistological study of 16 cases. Histopathology 1989;15:61-9.

17. Doglioni C, Dell'Orto P, Coggi G, Iuzzolino P, Bontempini L, Viale G. Choroid plexus tumors: an immunocytochemical study with particular reference to the coexpression of intermediate filament proteins. Am J Pathol 1987;127:519-29.

18. Furness PN, Lowe J, Tarrant GS. Subepithelial basement membrane deposition and intermediate filament expression in choroid plexus neoplasms and ependymomas. Histopathology 1990;16:251-5.

19. Kouno M, Kumanishi T, Washiyama K, Sekiguchi K, Saito T, Tanaka R. An immunohistochemical study of cytokeratin and glial fibrillary acidic protein in choroid plexus papilloma. Acta Neuropathol 1988;75:317-20.

20. Mannoji H, Becker LE. Ependymal and choroid plexus tumors: cytokeratin and GFAP expression. Cancer 1988;61: 1377-85.

21. Matsushima T, Inoue T, Takeshita I, Fukui M, Iwaki T, Kitamoto T. Choroid plexus papillomas: an immunohistochemical study with particular reference to the coexpression of prealbumin. Neurosurgery 1988;23:384-9.

22. Miettinen M, Clark R, Virtanen I. Intermediate filament proteins in choroid plexus and ependyma and their tumors. Am J Pathol 1986;123:231-40.

23. Paulus W, Jänisch W. Clinicopathologic correlations in epithelial choroid plexus neoplasms: a study of 52 cases. Acta Neuropathol 1990;80:635-41.

24. Rubinstein LJ, Brucher J-M. Focal ependymal differentiation in choroid plexus papillomas: an immunoperoxidase study. Acta Neuropathol 1981;53:29-33.

25. Taratuto AL, Molina H, Monges J. Choroid plexus tumors in infancy and childhood. Focal ependymal differentiation: an immunoperoxidase study. Acta Neuropathol 1983;59:304-8.

26. Gottschalk J, Jautzke G, Paulus W, Goebel S, Cervos-Navarro J. The use of immunomorphology to differentiate choroid plexus tumors from metastatic carcinomas. Cancer 1993;72: 1343-9. 
27. Kepes JJ, Collins J. Choroid plexus epithelium (normal and neoplastic) expresses synaptophysin. A potentially useful aid in differentiating carcinoma of the choroid plexus from metastatic papillary carcinomas. J Neuropathol Exp Neurol 1999; 58:398-401.

28. Albrecht S, Rouah E, Becker LE, Bruner J. Transthyretin immunoreactivity in choroid plexus neoplasms and brain metastases. Mod Pathol 1991;4:610-4.

29. Herbert J, Cavallaro T, Dwork AJ. A marker for primary choroid plexus neoplasms. Am J Pathol 1990;136:1317-25.

30. Loy TS, Calaluce RD. Utility of cytokeratin immunostaining in separating pulmonary adenocarcinomas from colonic adenocarcinomas. Am J Clin Pathol 1994;102:764-7.

31. Loy TS, Calaluce RD, Keeney GL. Cytokeratin immunostaining in differentiating primary ovarian carcinoma from metastatic colonic adenocarcinoma. Mod Pathol 1996;9:1040-4.

32. Wang NP, Zee S, Zarbo RJ, Bacchi CE, Gown AM. Coordinate expression of cytokeratins 7 and 20 defines unique subsets of carcinomas. Appl Immunohistochem 1995;3:99-107.

33. Perry A, Parisi JE, Kurtin PJ. Metastatic adenocarcinoma to the brain: an immunohistochemical approach. Hum Pathol
1997;28:938-43.

34. Burger PC, Scheithauer BW. Tumors of neuroglia and choroid plexus epithelium. Tumors of the central nervous system. Vol. 10. Washington, DC: Armed Forces Institute of Pathology; 1994. p. 136-42.

35. Bratthauer GL, Adams LR. Immunohistochemistry: antigen detection in tissue. In: Mikel UV, editor. Advanced laboratory methods in histology and pathology. Washington, DC: Armed Forces Institute of Pathology; 1994. p. 1-40.

36. Miettinen M. Keratin immunohistochemistry: update of applications and pitfalls. Pathol Annu 1993;28:113-43.

37. Ramaekers F, van Niekerk C, Poels L, Schaafsma E, Huijsmans A, Robben $\mathrm{H}$, et al. Use of monoclonal antibodies to keratin 7 in the differential diagnosis of adenocarcinomas. Am J Pathol 1990;136:641-55.

38. Miettinen M. Keratin 20: immunohistochemical marker for gastrointestinal, urothelial, and Merkel cell carcinomas. Mod Pathol 1995;8:384-8.

39. Moll R, Löwe A, Laufer J, Franke WW. Cytokeratin 20 in human carcinomas: a new histodiagnostic marker detected by monoclonal antibodies. Am J Pathol 1992;140:427-47.

\section{Book Review}

\section{Eissa S, Shoman S: Tumor Markers, 416 pp, London, Chapman and Hall Medical, 1998 (\$110).}

In a clear, well-organized, and authoritative manner, this book provides most of what the pathologist needs to know about tumor markers. The skills of Drs. Eissa and Shoman have come to the aid of many pathologists who are confronted with the difficulties of dealing with challenging tumors on a daily basis.

This book is composed of 10 chapters. The first chapter deals with technical aspects of immunohistochemistry, flow cytometry, and molecular biology techniques, which set the stage for the chapters that follow. Several chapters have been devoted to the more in-depth discussion of the role of tumor markers in diagnosis, tumor progression and proliferation, invasiveness and metastasis, response to therapy, and genetic analysis. The strongest section in this book is the section that deals with a large num- ber of tumors in selected organ systems. The editors' emphasis is to give readers good exposure to the different tumors in the particular organ; the histologic classification; criteria for diagnosis; and a large number of diagnostic, prognostic, and genetic biomarkers specific to that tumor. I found the text to be very informative and entertaining and most useful in my daily practice. The images are of high quality and are adequately labeled. The book is easy to navigate and is logical in its layout with an almost complete list of up-to-date references.

Overall, I found this book to be a must-have for pathology residents, fellows, and practicing pathologist. Other clinicians who have an interest in tumor biology and biomarkers will also find it to be of tremendous value.

\section{Ossama Tawfik \\ University of Kansas Medical Center \\ Kansas City, Kansas}

